\title{
Randomized, Double Blind and Placebo Controlled Study Using a Combination of Two Probiotic Lactobacilli to Alleviate Symptoms and Frequency of Common Cold
}

\author{
Regina Busch, Joerg Gruenwald, Steffi Dudek
}

Analyze \& Realize GmbH, Berlin, Germany.

Email: sdudek@analyze-realize.com

Received August $1^{\text {st }}, 2013$; revised September $1^{\text {st }}, 2013$; accepted September $9^{\text {th }}, 2013$

Copyright (C) 2013 Regina Busch et al. This is an open access article distributed under the Creative Commons Attribution License, which permits unrestricted use, distribution, and reproduction in any medium, provided the original work is properly cited.

\begin{abstract}
Purpose: The efficacy of ProbiDefendum, a combination of the two lactobacilli Lactobacillus plantarum HEAL9 and Lactobacillus paracasei 8700:2, on the severity, duration and frequency of cold episodes was studied in a randomized, double blind, placebo controlled clinical trial. Methods: A total of 310 subjects with increased risk for common cold infection (at least two episodes of common cold during the last six months) were enrolled and were randomly assigned to receive either active product (A-group) or placebo (P-group) over a period of 12 weeks. Subjects reported the occurrence and severity of cold episodes in a diary, scoring 13 different symptoms of common cold over a period of 7 days after the episode began. Results: In the total study population, cold episodes were reported in 148 cases (47.7\%). In the A-group, 54 subjects acquired one and 18 subjects acquired 2 episodes whereas 83 subjects had none. In the P-group, 52 subjects reported one, 24 subjects reported 2 episodes and 79 subjects reported none. Although the number of episodes was similar in both groups, cold episodes in the active group were significantly shorter than in the placebo group. The total sum scores of cold symptoms were significantly lower in the active group as compared with the placebo group, as well as in the intention-to-treat (ITT) as in the per-protocol (PP) collectives (ITT: $75.2 \pm 40.3$ vs. $113.4 \pm 66.3 ; \mathrm{p}<$ 0.001 ; PP: $73.1 \pm 38.8$ vs. $117.0 \pm 66.1 ; p<0.001$ ). The daily ingestion of the probiotics over a period of 12 weeks was well tolerated. Conclusion: The daily intake of the probiotic dietary supplement ProbiDefendum over a period of 12 weeks efficiently alleviated symptoms of common cold and the duration of cold episodes.
\end{abstract}

Keywords: Probiotics; Lactobacillus plantarum HEAL9; Lactobacillus paracasei 8700:2; Immune; Common cold; Clinical Study

\section{Introduction}

Common cold is an upper respiratory tract infection that can be caused by more than 100 different viruses. The most prominent of them is human rhinovirus (HRV), which itself comprises more than 150 serotypes [1]. The virus enters the human body through the mouth or the nose. Symptoms include sore throat, running nose, nasal congestion, sneezing and coughing, sometimes accompanied by headache and muscle aches. Until now, no efficient antiviral drugs are available and treatment options that only aim at alleviating specific symptoms. Episodes of common cold usually resolve within 7 days but longer episodes are observed as well.

Adults experience 2 - 5 cold episodes per year whereas children have 7 - 10 such viral infections [2]. Common cold is a substantial contributor to the number of sick days in schools, day care facilities and the work environment. In a Swedish survey from 2008, the total productivity loss in Sweden due to common cold infections was estimated to be about $€ 2.7$ billion per year, which is not only caused by the number of sick days, but also by low productivity while at work, and absenteeism of care givers. Reducing the lost productivity by 1 day per person and year in Sweden alone would potentially save more than $€ 500$ million. The survey also revealed that the productivity loss due to reduced working capacity of subjects at work having a common cold infection or allergenic rhinitis and losses because of absence from work are very similar [3]. Developing efficient treatment options for common cold is therefore of high public health 
interest and has high economic relevance. For infections like common cold, which are usually benign, consumers search for natural remedies that are supposed to be better tolerated than pharmaceutical products.

Non-pathogenic bacteria symbiotically living in the human intestine play a pivotal role in defending the organism from pathogenic microorganisms. During the last years, the efficacy of orally administered non-pathogenic bacteria (probiotics) for the prevention or treatment of common infectious diseases received considerable interest. The WHO defined probiotics as "live microorganisms which when administered in adequate amounts confer a health benefit to the host" [4]. Mechanisms by which probiotics exert their activities including the regulation of the intestinal microbial homeostasis, inhibition of colonization of pathogenic bacteria in the intestinal mucosa and modulation of the local and systemic immune defence. Most of the current applications of probiotics concern effects on the gastrointestinal system. A recent meta-analysis of 74 studies describing 84 trials and including all together 10,381 subjects revealed that probiotics can be beneficial for the treatment or prevention of several gastrointestinal diseases, such as pouchitis, infectious diarrhoea, irritable bowel syndrome, Helicobacter pylori, Clostridium difficile disease and antibiotic associated diarrhoea. The observed treatment effects are disease and strain specific and were not affected by the age of the subjects [5]. In a multicentre study by the European Society for Paediatric Gastroenterology, the administration of Lactobacillus GG in a rehydration solution resulted in a significant curtailment of episodes of viral diarrhoea in infants, whereas the efficacy of the test substance for the treatment of bacterial diarrhoea was inconclusive [6]. Several studies demonstrated that probiotics are efficient against upper respiratory tract infections [7-11]. Because probiotics may affect viral infections and exert their efficacy at extra-intestinal sites, immune-stimulation was suggested as contributor to the effects on respiratory tract infections [12]. Probiotic health effects are in general strain specific and cannot be extrapolated to other strains or other indications [13].

A combination of two probiotic lactobacilli, L. plantarum HEAL9 and L. paracasei 8700:2 has been shown to alleviate symptoms of common cold and to shorten the duration of common cold episodes in healthy adult subjects [11]. The aim of the present double blind, placebo controlled, randomized, clinical trial was to investigate the efficacy and safety of the same probiotic combination as the active study product in a population with increased risk of common cold (more than 2 episodes of common cold during the last 2 months). The primary endpoint of the study was the effect of the study product on the severity of cold symptoms whereas the effect on the fre- quency and duration of cold episodes were secondary endpoints.

\section{Material and Methods}

\subsection{Study Design}

The study was designed as a prospective, multicentre, randomized, double blind, placebo controlled trial, executed between January 2008 and June 2008 at four different study centres in the Berlin area, Germany. This nutritional study was performed according to the principles of the World Health Organization (Declaration of Helsinki/Hong Kong/Somerset) as well as the EU recommendations (CPMP/ICH/135/95). The subjects provided written informed consent. The study design, performance and maintenance of the study documentation complied with the GCP guidelines CPMP/ICH/135/95.

\subsection{Study Population}

The number of subjects needed for this study was determined by power analysis based on the results of a previous study with the same probiotic blend [11]. A total of 310 subjects were recruited, according to the following inclusion criteria: age $\geq 18$, at least 2 episodes of common cold in the last 6 months, and written consent to participate in the study. Exclusion criteria were active allergic rhinitis or asthma; known hypersensitivity to the ingredients of the investigational product; fever (body temperature $\geq 39^{\circ} \mathrm{C}$ ); severe organ or systemic disease; gastrointestinal disease; intake of immune system suppressants, immune system stimulants, analgesics/anti-rheumatics, anti-inflammatories, antitussives/expectorants, influenza medication, mouth or throat therapeutics; bacterial tonsillitis; cancer, HIV positive, pregnancy or nursing; alcohol abuse; medication or drug dependence; participation in a clinical trial within the last 30 days; or if compliance and completion of the subject's diary was not possible due to language difficulties.

The study population $(\mathrm{n}=310)$ was randomized by block randomization to take either the active substance (A-group, $\mathrm{n}=155$ ) or placebo (P-group, $\mathrm{n}=155$ ). They represent the "Full Analysis Set", corresponding to the "intention to treat" population (ITT). The population consisted of 206 females and 104 males $(66.5 \%$ and $33.5 \%$ resp).

There was no significant difference in the proportion of male/female subjects ( $p=0.470, \chi^{2}$ test) or in the age distribution between males and females $\left(p_{u}=0.540\right)$ between the active and the placebo group. The average age was $46.0 \pm 17.1$ (range $18-88$ years). At enrolment, 116 out of 310 subjects (37.4\%) were not older than 40 years of age. 305 subjects were classified as Caucasian, 2 were of Asian descent, and 3 subjects could not be assigned to 
a specific ethnic group.

There were no statistically significant differences between the active and the placebo group with respect to age, gender or anthropometric data (Table 1).

\subsection{Interventions}

The probiotic used in the present study was a blend of Lactobacillus plantarum HEAL 9 (DSM 15312) and Lactobacillus paracasei 8700:2 (DSM 13434), purchased from DSM.

The subjects were provided with pre-numbered, identical-looking one-serving sachets of $1 \mathrm{~g}$ each, containing either investigational product or placebo (84 sachets per subject). The test product contained $1 \times 10^{9} \mathrm{cfu} / \mathrm{g}$ of the probiotic blend and the excipient maltodextrin whereas the placebo consisted of maltodextrin alone. The sachets were provided by the sponsor, Probi AB.

Subjects were instructed to dissolve one sachet in 200 $\mathrm{ml}$ water and to ingest one prepared drink per day with a meal over a period of 12 weeks, or until at least the 7 th day into the second cold episode.

\subsection{Outcome Measures}

The primary endpoint of this study was to examine whether the daily intake of $1 \mathrm{~g}$ of the probiotic product composed of $L$. plantarum HEAL 9 and $L$. paracasei $8700: 2$ significantly reduced the total sum score of symptoms (TS) of common cold as compared with placebo. The total sum scores were calculated from the patient diaries that the subjects were instructed to keep for seven days after the onset of a cold episode.

In these diaries, subjects evaluated 13 of the most common symptoms of cold on a 4-point rating scale as follows: complaint free $=0$, slight $=1$, moderate $=2$, severe $=3$ resulting in an individual symptom score (Table 2).

Adding together all individual symptom scores for a particular day resulted in the total daily sum score (TDS),

Table 1. Characteristics of the participants at inclusion.

\begin{tabular}{cccc}
\hline & $\begin{array}{c}\text { P-group } \\
(\mathbf{n}=\mathbf{1 5 1})\end{array}$ & $\begin{array}{c}\text { V-group } \\
(\mathbf{n}=\mathbf{1 5 5})\end{array}$ & p-value \\
\hline & $\begin{array}{c}\text { Mean } \pm \text { SD } \\
\text { (Range) }\end{array}$ & $\begin{array}{c}\text { Mean } \pm \text { SD } \\
(\text { Range) }\end{array}$ & \\
& $46.6 \pm 17.2$ & $45.3 \pm 17.1$ & 0.581 \\
Age (years) & $(18-87)$ & $(18-88)$ & \\
& $49 / 106$ & $55 / 100$ & \\
Sex (m/f) & $170.8 \pm 8.4$ & $171.1 \pm 7.9$ & 0.523 \\
Body height $(\mathrm{cm})$ & $(150-192)$ & $(155-191)$ & \\
& $71.4 \pm 13.1$ & $72.5 \pm 13.1$ & 0.512 \\
Body weight $(\mathrm{kg})$ & $(48-120)$ & $(50-120)$ & \\
& $24.5 \pm 4.3$ & $24.7 \pm 3.8$ & 0.331 \\
BMI $\left(\mathrm{kg} / \mathrm{cm}^{2}\right)$ & $(17.4-42.5)$ & $(18.0-37.9)$ & \\
& & & \\
\hline
\end{tabular}

Table 2. Schematic presentation of the patient diary assessing the severity of symptoms during the cold episodes.

\begin{tabular}{|c|c|c|c|c|}
\hline & \multicolumn{4}{|c|}{ Score } \\
\hline & $\mathbf{0}$ & 1 & 2 & 3 \\
\hline General symptoms: & & & & \\
\hline -Headache & & & & \\
\hline -Pains in the limbs & & & & \\
\hline Pharyngeal symptoms: & & & & \\
\hline -Sore throat & & & & \\
\hline -Difficulty swallowing & & & & \\
\hline -Hoarseness & & & & \\
\hline Nasal symptoms: & & & & \\
\hline -Runny nose & & & & \\
\hline -Congested nose & & & & \\
\hline -Yellow secretion & & & & \\
\hline -Bloody secretion & & & & \\
\hline -Sneezing & & & & \\
\hline Bronchial symptoms: & & & & \\
\hline- Cough & & & & \\
\hline -Yellow secretion of the bronchial tubes & & & & \\
\hline -Other secretion & & & & \\
\hline
\end{tabular}

which could reach a maximum of 39 per subject/day $(3 \times$ 13 for the highest severity in all 13 points). The sum over all 7 or 14 TDS' (one or two episodes, resp.) is the total sum score of symptoms (TS). The TS per subject for the entire study can be as high as $546(39 \times 7 \times 2)$ if two episodes of common cold occurred.

During the study period, 2 regular visits were scheduled with up to 2 more visits in case of one or two cold episodes. At the first visit, the investigator assessed the baseline data such as body weight, heart rate, blood pressure and body temperature, and concurrent medications and therapies. In case a cold episode occurred, subjects were instructed to present themselves to the investigator for confirmation of the episode, to keep a symptom diary for the 7 days following the first symptoms of an episode, and to contact the investigator by phone after 7 days to verify the symptoms. Subjects who experienced two cold episodes visited the investigator 7 days after the symptoms of the second episode had began. During each visit, adverse events were recorded. At the end of the study, the remaining sachets were returned and both the investigator and the subject appraised the global efficacy and safety of the products.

Compliance with respect to the indicated daily dose of test substance or placebo was determined by counting the unused sachets returned at the last visit and relating them to the days the subject participated in the study. Subjects whose intake deviated by more than $20 \%$ from the protocol (e.g. $>20 \%$ of sachets too much or less returned) were considered non-compliances and excluded from the PP collective.

The secondary endpoints of this study were the changes in frequency and duration of cold episodes and a global evaluation of the efficacy of the study product made by the investigators and the subjects in the cases where cold episodes occurred. 


\subsection{Statistical Methods}

The hypothesis that the total sum score for cold symptoms in the active group is significantly lower than in the placebo group was tested against the null-hypothesis that both are the same using a non-parametric Mann-Whitney-U-test ( $\mathrm{p}_{\mathrm{u}}$-value). Where appropriate, F-, t-, and $\chi^{2}$-tests were performed for the full analysis set (ITT) and the valid case analysis set (PP). p-values $>0.05$ are considered statistically not significant, whereas $p<0.05$ indicates statistical significance.

\section{Results}

\subsection{Compliance}

A total of 310 subjects with increased susceptibility to common cold infections were enrolled and randomized to receive the test substance or placebo. These 310 subjects represent the population for the Full Analysis Set (FAS), which corresponds to the Intention-to-Treat population (ITT). Of these 310 subjects, 303 finished the study.

A total of 26 subjects $(8.4 \%)$ were considered as noncompliant and were excluded from the PP collective (PP: $\mathrm{n}=284,91.6 \%)$. Non-compliances concerned early termination of the study, incomplete diaries, or deviations from the per-protocol use of the test substances by more than $20 \%( \pm)$. Figure 1 illustrates the ITT and PP study populations.

\subsection{Incidence of Infections}

In the ITT collective, 148 subjects (76 in the P-group, 72 in the A-group) experienced at least one episode of common cold during the study period. Of these, 42 subjects (24 P-group, 18 A-group) experienced 2 episodes.

There was no statistically significant difference between the active and the placebo group (Table 3).

Analysing the PP population confirmed the result (Table 4). The daily ingestion of $1 \mathrm{~g}$ of a blend of the probiotics Lactobacillus plantarum and L. paracasei over a period of 12 weeks did not reduce the number of cold episodes. The time to the onset of the first episode or the time between the episodes was similar in the active and the placebo group (data not shown).

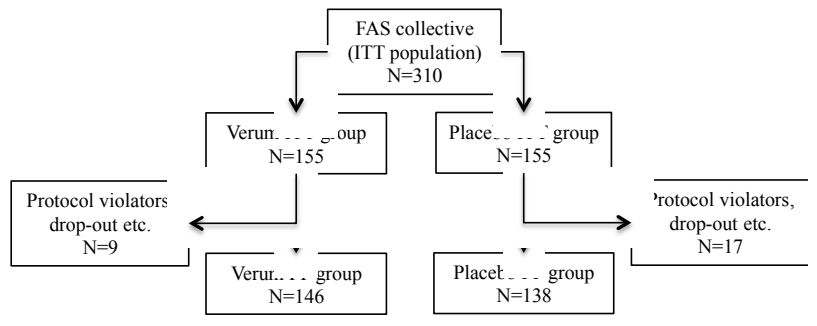

Figure 1. Schematic representation of the study population. ITT: intention to treat, PP: per protocol.
Table 3. Frequency of common cold events in the intention-to-treat (ITT) population.

\begin{tabular}{ccccc}
\hline $\begin{array}{c}\text { Number of } \\
\text { Episodes }\end{array}$ & $\begin{array}{c}\text { Total } \\
(\mathbf{n = 3 1 0 )}\end{array}$ & $\begin{array}{c}\text { P-group } \\
(\mathbf{n}=\mathbf{1 5 5})\end{array}$ & $\begin{array}{c}\text { V-group } \\
(\mathbf{n}=\mathbf{1 5 5})\end{array}$ & $\begin{array}{c}\text { p-value } \\
\left(\chi^{2} \text {-test) }\right.\end{array}$ \\
\hline \multirow{n}{*}{$\mathbf{n}(\mathbf{\%})$} & $\mathbf{n}(\%)$ & $\mathbf{n ~ ( \% )}$ & \\
$\geqq 1$ & $162(52.3 \%)$ & $79(51.0 \%)$ & $83(53.5 \%)$ & 0.649 \\
1 & $106(47.7 \%)$ & $76(49.0 \%)$ & $72(46.5 \%)$ & \\
2 & $42(13.5 \%)$ & $52(33.5 \%)$ & $54(34.8 \%)$ & 0.811 \\
\hline
\end{tabular}

Table 4. Frequency of common cold events in the per-protocol (PP) population.

\begin{tabular}{ccccc}
\hline $\begin{array}{c}\text { Number of } \\
\text { Episodes }\end{array}$ & $\begin{array}{c}\text { Total } \\
(\mathbf{n = ~ 2 8 4 )}\end{array}$ & $\begin{array}{c}\text { P-group } \\
(\mathbf{n = 1 3 8})\end{array}$ & $\begin{array}{c}\text { V-group } \\
(\mathbf{n = 1 4 6})\end{array}$ & $\begin{array}{c}\text { p-value } \\
\left(\chi^{2} \text {-test) }\right.\end{array}$ \\
\hline None & $\mathbf{n ~ ( \% )}$ & $\mathbf{n ~ ( \% )}$ & $\mathbf{n ~ ( \% )}$ & \\
$\geqq 1$ & $146(51.4 \%)$ & $68(49.3 \%)$ & $78(53,4 \%)$ & 0.484 \\
1 & $101(35.6 \%)$ & $50(36.2 \%)$ & $51(34.9 \%)$ & 0.817 \\
2 & $37(13.0 \%)$ & $20(14.5 \%)$ & $17(11.6 \%)$ & 0.576 \\
\hline
\end{tabular}

\subsection{Reduction of the Sum Score of Cold Symptoms}

The primary target criterion of this nutritional study was to prove that the ingestion of the combination of two probiotic lactobacillus strains (L. planatrum HEAL9 and L. paracasei 8700:2) over a period of three months significantly reduced the total sum score of symptoms (TS) of common cold as compared with placebo. The TS score is the sum of all total daily sum scores of symptoms (TDS), which in turn are calculated from the patient diaries, scoring 13 relevant cold symptoms on a scale from 0 to 3 (Table 2). The TS calculation was only possible when the diaries were complete. Three subjects in each group did not provide complete diaries and were excluded from the calculation of the TS. Therefore, the number of subjects in the ITT population suitable for the TS calculation, who experienced at least one cold episode dropped from 148 to 142 (A-group: $\mathrm{n}=68$, P-group: $\mathrm{n}=74$ ). Within the eligible ITT population, ingesting the probiotic product significantly reduced the TS in the active group as compared with the placebo group. In the PP collective, a similar picture was obtained. If only subjects treated according to protocol were taken into account, a statistically significant difference between the total sum scores for active and placebo resulted (Table 5).

The question arose whether the TDS scores in the active group were consistently improved over the course of the episode. It was also important to exclude that different scores at the beginning of the episodes had confounded the results.

As illustrated in Table 6, the TDS values at the first day of all episodes are similar, suggesting that the sever- 
Table 5. Mean total sum scores of cold symptoms (TS) for the ITT and PP collectives during the entire study period.

\begin{tabular}{ccccccc}
\hline & \multicolumn{3}{c}{ ITT population } & \multicolumn{3}{c}{ PP population } \\
\hline TS Score (points) & n & Mean & SD & n & Mean & SD \\
Total & 142 & 95.1 & 58.4 & 134 & 95.7 & 58.7 \\
P-group & 74 & 113.4 & 66.3 & 69 & 117.0 & 66.1 \\
V-group & 68 & 75.2 & 40.3 & 69 & 73.1 & 38.8 \\
p-value (t-Test) & \multicolumn{3}{c}{$<0.001^{*}$} & & \multicolumn{4}{c}{$<0.001^{*}$} \\
\hline
\end{tabular}

Table 6. Mean total daily sum scores of cold symptoms (TDS) for ITT subjects on day 1 of the first and second episodes.

\begin{tabular}{ccccccc}
\hline & \multicolumn{3}{c}{ Day $\mathbf{1} / \mathbf{1}^{\text {st }}$ episode } & \multicolumn{3}{c}{ Day $\mathbf{1} / \mathbf{2}^{\text {nd }}$ episode } \\
\hline TS Score & $\mathbf{n}$ & Mean & SD & $\mathbf{n}$ & Mean & SD \\
Total & 142 & 18.7 & 9.9 & 39 & 16.7 & 9 \\
P-group & 74 & 18.7 & 10.8 & 22 & 17.2 & 10.2 \\
V-group & 68 & 18.6 & 9 & 17 & 16.1 & 7.3 \\
p-value & & 0.979 & & & 0.707 & \\
\hline
\end{tabular}

ity of the cold symptoms was comparable in all cases.

The TDS values for all 185 episodes in the PP group were plotted over the course of 7 days (Figure 2). The graph illustrates that daily symptom scores in the active group are consistently lower than in the placebo group.

This difference becomes statistically significant on day 4. The primary endpoint of the study, the reduction of the severity of common cold symptoms by the ingestion of the probiotic product was met.

\subsection{Duration of the Episodes}

The duration of an episode is defined as the number of days during an episode that the subject is not symptom-free.

Whereas episodes in the placebo-group lasted for an average of 6.7 days, subjects in the active group were symptom free after 5.6 days, more than one day faster than in the placebo group (Table 7). Subjects who ingested the probiotic product during the cold episode apparently recovered faster than subjects taking placebo.

As already shown in Table 3, the number of subjects experiencing a second episode was similar in both groups. If a second episode occurred, the period between the episodes was not different for the placebo and the active group (data not shown).

\subsection{Safety of the Intervention}

The safety of the test substance was investigated during each visit at the study centres. Neither the global safety evaluation by the investigators nor subjects revealed differences between placebo and test substance. In more than $95 \%$ of the cases, the safety of both products was

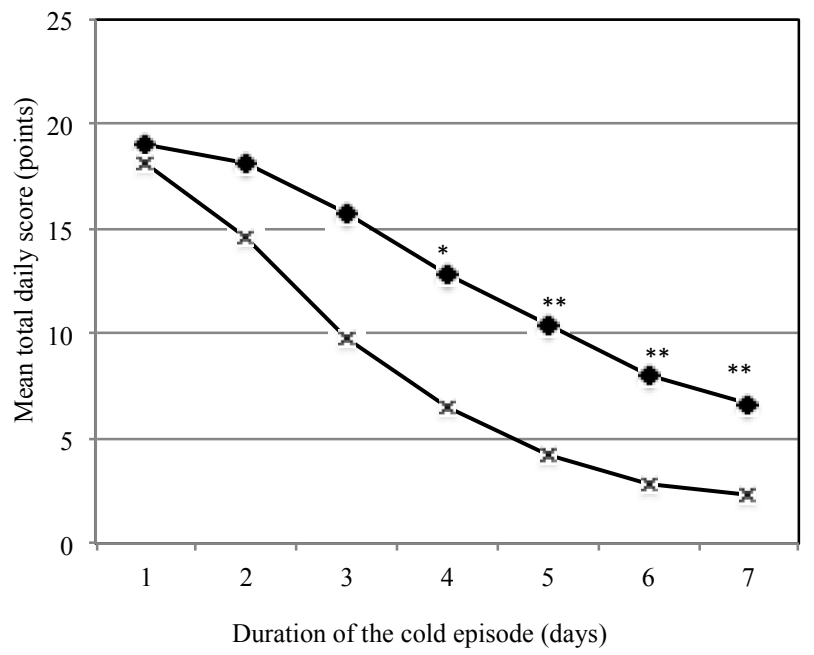

Figure 2. Development of the mean total daily scores (TDS) of cold symptoms over the duration of one episode. Placebo $(\mathrm{n}=90)$, Active group $(\mathrm{n}=\mathbf{8 2}),{ }^{*} \mathrm{p}=\mathbf{0 . 0 3 4},{ }^{* * *} \mathrm{p}<$ 0.001.

Table 7. Duration of cold episodes (= days with cold symptoms).

\begin{tabular}{cccc}
\hline & \multicolumn{3}{c}{ Duration of episodes [days] } \\
\hline & $\mathbf{n}$ & Mean & SD \\
\hline Total & 182 & 6.2 & 1.2 \\
P-group & 98 & 6.7 & 0.8 \\
V-group & 87 & 5.6 & 1.3 \\
p-value (t-Test) & & $<0.001^{*}$ \\
\hline
\end{tabular}

considered very good or good, both by the investigator and the subject.

No clinically relevant changes in the clinical parameters body weight, heart rate, systolic and diastolic blood pressure or body temperature between start and the end of the study were observed.

Adverse events were documented in 19 cases out of the ITT population of 310 subjects (10 A-group, 9 Pgroup). The differences between the numbers of adverse events in both groups were statistically not significant.

Documented adverse events included accidental trauma $(\mathrm{n}=4)$, diarrhoea $(\mathrm{n}=2)$, whiplash trauma $(\mathrm{n}=$ $1)$; conjunctivitis $(\mathrm{n}=1)$; inflammation of the bladder ( $\mathrm{n}$ $=2)$; headache (1); tonsillitis $(\mathrm{n}=2)$; backache $(\mathrm{n}=2)$; epicondilitis $(\mathrm{n}=1)$; abdominal pain $(\mathrm{n}=2)$, dizziness and fatigue (1) and 1 pregnancy. Five of the adverse events (3 A-group, 2 P-group) let to premature termination of the study (tonsillitis (2 A-group, with strong intensity), diarrhea (1 A-group, with moderate intensity but persistent), abdominal pain (1 P-group, with strong intensity), pregnancy (1 P-group)), The remaining adverse events were considered not to be severe by the investigators and the study was continued. For all but one ad- 
verse event (moderate but persistent diarrhoea) a relation to the intervention could be ruled out.

\section{Discussion}

There is increasing evidence that probiotics, although being primarily active in the gastrointestinal tract, may exert health effects in other organs as well. Probiotic and conventional lactobacilli have been shown to stimulate the immune system, thereby alleviating or preventing microbial infections in other organs than the intestinal tract, where their effect is traditionally expected [14]. The postulated immune-stimulatory effect triggered the interest by the food industry and motivated numerous human intervention trials on different aspects of immunity.

Until now, the evidence that the administration of probiotics is beneficial for the prevention or alleviation of viral upper respiratory tract infections is still not fully conclusive. Besides the limited number of adequate RCT studies, their outcomes are rarely comparable due to differences in study design, species and strains of the probiotics tested and study populations used. Moreover, some methodological bias may affect the interpretation of probiotic studies [15].

The study presented here is the second study with a combination of Lactobacullus plantarum HEAL 9 (DSM 15312) and L. paracasei 8700:2 (DSM 13434). The study designs were similar, although the primary endpoints were different and the two studies were performed at different occasions (2007 and 2008), at different locations (Sweden and Germany) and by different CROs. The seasonal pattern of common cold infections was accounted for as both were executed between January and April, the peak time of rhinovirus infections. The previous study was performed with healthy adults without further selection for infection risk [11], whereas only subjects with a slightly higher risk for common cold infections were enrolled in the present study. In the present study, in order to limit the possible bias by self-reporting, the investigators verified each infection during the visits (beginning of each episode) and by phone contact with the subjects (at the end of the episodes), which certainly increased the reliability of the data. Since no markers correlating with the incidence and severity of viral respiratory infections have been found so far, no further biochemical analysis was performed.

The present study, although performed under different conditions, confirmed the findings of the previous one that the severity and duration of common cold episodes are reduced upon ingestion of the specific probiotic product. Symptoms of cold were alleviated throughout the entire cold episodes, which in turn affected the duration of the episodes. In both studies, the daily ingestion of the probiotic lactobacilli significantly shortened the duration of cold episodes by approximately $15 \%$, from 8.6 days to 7.2 days $(p<0.05)$ in the first study [11], and from 6.7 days to 5.6 days $(\mathrm{p}<0.001)$ in the present study.

Both studies demonstrated that ingesting the probiotic test substance reduced the severity of cold episodes, as represented by the total sum scores of symptoms but the effect was more pronounced in the present study as compared with the first one. Although the total sum score in the first study was lower in the probiotic group as compared with placebo, significance was only reached for pharyngeal symptoms. In the study presented here, the difference was significant both for the total sum score and for most of the individual symptom scores, except for some bronchial symptoms.

The present study did not confirm the finding of the first study that the ingestion of probiotics reduces the incidence of cold episodes and may postpone the onset of subsequent episodes. In the first study, $67 \%$ in the placebo group acquired one or more cold episodes $(\mathrm{p}=$ 0.043 ), but only $55 \%$ of the subjects in the active group. The difference increased even further for recurrent cold episodes, which were reported by $33 \%$ of the subjects in the placebo group but by only $21 \%$ in the active group (p $=0.024)$. In the present study, the incidence of cold episodes was similar in both groups $(49 \%$ in the placebo and $46.3 \%$ in the active group for the ITT collective, $50.7 \%$ and $46.6 \%$ for placebo and active group respectively in the PP population).

Selecting healthy subjects with a slightly higher risk for common cold infection did not result in a higher total number of cold episodes in the present study but may have had an impact on the efficacy on the common cold incidence. Selecting study subjects based on self-reported past infection frequency appears to be inefficient to compensate for the natural dynamic of seasonal respiratory viruses and to increase the number of episodes in the study population.

The results of both studies are in line with previous findings with 3 different probiotic bacteria in combination with a multivitamin/mineral blend, showing a reduced severity of symptoms and shorter cold episodes but no effect on incidence of infections. In a RCT study in Germany with 477 adult subjects, a probiotic mix consisting of Lactobacillus gasseri PA 16/8, Bifidobacterium longum SP07/3 and B. bifidum MF $25 / 5$ was administered in combination with minerals and vitamins [9]. A similar study on 479 subjects with the same probiotics in 10-times lower concentration confirmed the clinical findings. Duration and severity of cold episodes were improved but the incidence of infections was not affected by the supplement [16]. Both studies provide consistent 
evidence that the ingestion of these three probiotic bacteria strains in combination with a multivitamin-mineral mix affects the course of common cold episodes, although an impact of the vitamins and minerals on the outcome cannot be excluded.

The outcome of the present study also confirms earlier observations. The impact of Lactobacillus GG (LGG) on the alleviation of common cold was investigated in a study with 571 children at 18 different day care centres in Helsinki, Finland [17]. LGG ingestion over a longer period of time reduced the number of sick days, the severity of symptoms and the prescription of antibiotic treatments for respiratory infections. The treatment did not affect the incidence of infection.

It is a limitation of the study that the subjective assessment of the severity of symptoms was not accompanied by biochemical analysis or the identification of the type of infection.

The development of cold symptoms is considered an inflammatory response of the host to the viral challenge. More systematic data on appropriate biochemical markers are needed to establish an association between the inflammatory response to the viral infection and the beneficial physiological effects assigned to probiotics. Experts consider such a cause and effect relationship a precondition for the substantiation of an immune-related health claim [18]. Despite considerable efforts by many groups, a clear association between immunological or biochemical parameters and the reported pattern or intensity of cold symptoms has not been established yet [19]. Although laboratory parameters were not analysed, the present study provided valuable information to elucidate the aspects of viral infections that respond to the long-term ingestion of probiotics. The reduced intensity of symptoms and shortened length of the episodes point towards an immune-stimulatory effect of probiotics that helps the body cope with the infection rather than a direct interaction with the viral pathogens.

\section{Conclusion}

The daily ingestion by healthy adult subjects with a slightly increased risk of common cold of a combination of Lactobacillus plantarum HEAL 9 and L. paracasei $8700: 2$ over a period of 12 weeks alleviated the severity of common cold and shortened the duration of cold episodes by more than 1 day. The incidence of common cold infection was not affected by the lactobacilli in this population.

\section{Acknowledgements}

The study was a commission by Probi AB, Lund, Sweden. The authors thank Norman Bitterlich, PhD, for sup- porting the statistical analysis, and Mr. Niklas Larson, $\mathrm{PhD}$, for the critical review of the paper.

\section{REFERENCES}

[1] J. E. Linder, et al., "Human Rhinovirus C: Age, Season, and Lower Respiratory Illness over the Past 3 Decades," The Journal of allergy and clinical immunology, Vol. 131, No. 1, 2013, pp. 69-77,e61-66.

[2] R. Eccles, "Understanding the Symptoms of the Common Cold and Influenza," The Lancet Infectious Diseases, Vol. 5, No. 11, 2005, pp. 718-725. http://dx.doi.org/10.1016/S1473-3099(05)70270-X

[3] J. Hellgren, A. Cervin, S. Nordling, A. Bergman and L. O. Cardell, "Allergic Rhinitis and the Common Cold-High Cost to Society," Allergy, Vol. 65, No. 6, 2010, pp. 776783. http://dx.doi.org/10.1111/j.1398-9995.2009.02269.x

[4] "Health and Nutritional Properties of Probiotics in Food Including Powder Milk with Live Lactic Acid Bacteria," Joint FAO/WHO Expert Consultation on Evaluation of Health and Nutritional Properties of Probiotics in Food including Powder Milk with Live Lactic Acid Bacteria, Cordoba, October 1-4, 2001, pp. 1-34.

[5] M. L. Ritchie and T. N. Romanuk, "A Meta-Analysis of Probiotic Efficacy for Gastrointestinal Diseases," PLoS One, Vol. 7, No. 4, 2012, Article ID: e34938. http://dx.doi.org/10.1371/journal.pone.0034938

[6] S. Guandalini, et al., "Lactobacillus GG Administered in Oral Rehydration Solution to Children with Acute Diarrhea: a Multicenter European Trial," Journal of Pediatric Gastroenterology and Nutrition, Vol. 30, No. 1, 2000, pp. 54-60. http://dx.doi.org/10.1097/00005176-200001000-00018

[7] K. Hatakka, "Probiotics and the Prevention of Clinical Manifestation of Common Infectious Diseases in Children and in the Elderly," Ph.D. Thesis, Helsinki Univerity, Helsinki, 2007.

[8] M. de Vrese, et al., "Probiotic Bacteria Reduced Duration and Severity but not the Incidence of Common Cold Episodes in a Double Blind, Randomized, Controlled Trial," Vaccine, Vol. 24, No. 44-46, 2006, pp. 6670-6674. http://dx.doi.org/10.1016/j.vaccine.2006.05.048

[9] P. Winkler, M. de Vrese, C. Laue and J. Schrezenmeir, "Effect of a Dietary Supplement Containing Probiotic Bacteria plus Vitamins and Minerals on Common Cold Infections and Cellular Immune Parameters," International Journal of Clinical Pharmacology and Therapeutics, Vol. 43, No. 7, 2005, pp. 318-326. http://dx.doi.org/10.5414/CPP43318

[10] G. J. Leyer, S. Li, M. E. Mubasher, C. Reifer and A. C. Ouwehand, "Probiotic Effects on Cold and InfluenzaLike Symptom Incidence and Duration in Children," Pediatrics, Vol. 124, No. 2, 2009, pp. e172-179. http://dx.doi.org/10.1542/peds.2008-2666

[11] A. Berggren, I. Lazou Ahren, N. Larsson and G. Onning, "Randomised, Double-blind and Placebo-Controlled Study Using New Probiotic Lactobacilli for Strengthening the Body Immune Defence Against Viral Infections," Eu- 
ropean Journal of Nutrition, Vol. 50, No. 3, 2011, pp. 203-210. http://dx.doi.org/10.1007/s00394-010-0127-6

[12] H. Gill and J. Prasad, "Probiotics, Immunomodulation, and Health Benefits," In: Z. Bösze, Ed., Bioactive Components of Milk, Springer, New York, 2008, pp. 423-454. http://dx.doi.org/10.1007/978-0-387-74087-4_17

[13] F. Guarner, T. Requena and A. Marcos, "Consensus Statements from the Workshop Probiotics and Health: Scientific evidence," Nutricion Hospitalaria, Vol. 25, No. 5, 2010, pp. 700-704.

[14] M. de Vrese and J. Schrezenmeir, "Probiotics and NonIntestinal Infectious Conditions," The British Journal of Nutrition, Vol. 88, Suppl. 1, 2002, pp. S59-S66.

[15] R. L. Koretz, "Probiotics, Critical Illness, and Methodologic Bias," Nutrition in Clinical Practice, Vol. 24, No. 1, 2009, pp. 45-49. http://dx.doi.org/10.1177/0884533608329296

[16] M. de Vrese, et al., "Effect of Lactobacillus gasseri PA 16/8, Bifidobacterium longum SP 07/3, B. bifidum MF
20/5 on Common Cold Episodes: A Double Blind, Randomized, Controlled Trial," Clinical Nutrition, Vol. 24, No. 4, 2005, pp. 481-491.

http://dx.doi.org/10.1016/j.clnu.2005.02.006

[17] K. Hatakka, et al., "Effect of Long Term Consumption of Probiotic Milk on Infections in Children Attending Day Care Centres: Double Blind, Randomised Trial," BMJ, Vol. 322, No. 7298, 2001, p. 1327. http://dx.doi.org/10.1136/bmj.322.7298.1327

[18] EFSA Panel on Dietetic Products Nutrition, "Guidance on the Scientific Requirements for Health Claims Related to Gut and Immune Function," EFSA Journal, Vol. 9, No. 4, 2011, p. 1984.

[19] B. Barrett, R. Brown, R. Voland, R. Maberry and R. Turner, "Relations among Questionnaire and Laboratory Measures of Rhinovirus Infection," The European Respiratory Journal, Vol. 28, No. 2, 2006, pp. 358-363. http://dx.doi.org/10.1183/09031936.06.00002606

\author{
Abbreviations \\ HVR: human rhinovirus; \\ ITT: intention to treat; \\ PP: per protocol; \\ TDS: total daily score of symptoms; \\ TS: total sum score of symptoms.
}

